\title{
Laparoscopic adrenalectomy: comparison of outcomes between posterior retroperitoneoscopic and transperitoneal adrenalectomy with 10 years' experience
}

\author{
Yoonwon Kook $^{1 \#}$, Hye Ryeon Choi ${ }^{2 \#}$, Sang-Wook Kang ${ }^{1}$, Jin Kyong Kim ${ }^{1}$, Cho Rok Lee ${ }^{1}$, Jandee Lee ${ }^{1}$, \\ Jong Ju Jeong ${ }^{1}$, Kee-Hyun Nam ${ }^{1}$, Woong Youn Chung ${ }^{1}$ \\ ${ }^{1}$ Department of Surgery, Yonsei University College of Medicine, Seoul, Korea; ${ }^{2}$ Department of Surgery, Eulji Medical Center, Seoul, Korea \\ Contributions: (I) Conception and design: SW Kang; (II) Administrative support: SW Kang; (III) Provision of study materials or patients: JK Kim, CR \\ Lee, J Lee, JJ Jeong, KH Nam, WY Chung, SW Kang; (IV) Collection and assembly of data: Y Kook, HR Choi; (V) Data analysis and interpretation: \\ Y Kook, HR Choi; (VI) Manuscript writing: All authors; (VII) Final approval of manuscript: All authors. \\ \#These authors contributed equally to this work. \\ Correspondence to: Sang-Wook Kang, MD. Department of Surgery, Yonsei University College of Medicine, 50-1 Yonsei-ro, Seodaemun-gu, 03722, \\ Seoul, Korea. Email: oralvanco@yuhs.ac.
}

\begin{abstract}
Background Laparoscopic adrenalectomy is widely used for treating various adrenal tumors. Posterior retroperitoneoscopic adrenalectomy was introduced after transperitoneal laparoscopic adrenalectomy. The comparability and superiority of posterior retroperitoneoscopic adrenalectomy have been widely investigated. We aimed to compare the outcomes of posterior retroperitoneoscopic adrenalectomy and transperitoneal laparoscopic adrenalectomy using 10 years' data. The changes in outcomes over time were also analyzed.

Methods: This was a retrospective observational study. A total of 505 patients who underwent laparoscopic adrenalectomy between 2009 and 2018 were included. The patients were divided into two groups: transperitoneal $(n=114)$ and retroperitoneal $(n=391)$ groups. Patients who underwent posterior retroperitoneoscopic adrenalectomy were further classified into sub-groups (sub-group 1: 2009-2012; subgroup 2: 2013-2015; and sub-group 3: 2016-2018) based on time periods. Clinicopathological factors and postoperative outcomes were retrospectively reviewed and analyzed.

Results: The retroperitoneal group showed significantly shorter operation time, diet initiation time, and hospital stay compared to the transperitoneal group ( $\mathrm{P}=0.000,0.000$ and 0.000 , respectively). Analgesics were used less frequently in the retroperitoneal group than in the transperitoneal group $(\mathrm{P}=0.048)$. In the retroperitoneal group, the time to postoperative diet initiation shortened over time $(\mathrm{P}=0.000)$.

Conclusions: Posterior retroperitoneoscopic adrenalectomy is a favorable surgical method that results in patient outcomes that are comparable to those of conventional transperitoneal laparoscopic adrenalectomy, without compromising patient safety.
\end{abstract}

Keywords: Posterior retroperitoneoscopic adrenalectomy; laparoscopy; minimally invasive surgery; transperitoneal adrenalectomy

Submitted Mar 18, 2021. Accepted for publication Jun 17, 2021.

doi: $10.21037 /$ gs-21-178

View this article at: https://dx.doi.org/10.21037/gs-21-178

^ ORCID: Yoonwon Kook, 0000-0002-0756-738x; Hye Ryeon Choi, 0000-0002-5657-5677. 


\section{Introduction}

Adrenalectomy is indicated for various pathologic conditions of the adrenal glands. Since minimally invasive adrenalectomy using laparoscopy was introduced in 1992 by Gagner et al. (1), its feasibility has been investigated widely (2-5). Its advantages over open adrenalectomy include less postoperative pain and ileus, faster convalescence, and better cosmesis; hence, laparoscopic adrenalectomy has become the gold standard of treatment for most benign adrenal masses and even small malignant tumors $(6,7)$. The transperitoneal method is used conventionally to approach the adrenal glands, which allows for a familiar and large operating field (8). Based on the retroperitoneal location of the adrenal glands, Walz et al. introduced posterior retroperitoneoscopic adrenalectomy (PRA), which has been reported as a safe and effective technique (9). Many authors have compared laparoscopic transperitoneal adrenalectomy (TPA) and PRA and reported comparable or superior outcomes with PRA. In addition, PRA permits direct visualization of the adrenal glands without mobilizing adjacent organs $(8,10,11)$. In South Korea, the first successful PRA was performed in Yonsei university medical center in 2009; subsequently, the center adopted the method and reported the feasibility of PRA compared to that of TPA (12). As the institution that first started PRA in South Korea, we summarized the results of laparoscopic adrenalectomy performed over the past 10 years in our center. We also analyzed the changes in PRA outcomes over time. We present the following article in accordance with the STROBE reporting checklist (available at https:// dx.doi.org/10.21037/gs-21-178).

\section{Methods}

This was a retrospective observational study. Data of patients $(n=580)$ who underwent laparoscopic adrenalectomy from January 2009 to June 2018 were collected. After the exclusion of cases involving concomitant operations $(n=56)$, bilateral adrenalectomy $(n=12)$, secondary operations in patients with a history of adrenalectomy $(n=3)$, robotassisted surgery $(n=3)$, and non-minimally invasive open adrenalectomy $(\mathrm{n}=1), 505$ patients who underwent minimally invasive adrenalectomy were included (Figure 1).

The patients were divided into two groups based on the approach used: transperitoneal (TPA, $n=114)$ and retroperitoneal (PRA, n=391) groups. Patients who underwent PRA were further classified into three sub- groups based on time periods to determine if the outcomes changed over time. Clinicopathological factors, operative time, and postoperative outcomes including estimated blood loss, timing of postoperative diet initiation, postoperative hospital stay, and postoperative analgesic use were analyzed. Changes in surgical case selection over time in PRA group were also analyzed.

TPA was first performed in 2001 in our center. Since the first PRA was performed in 2009, it has become the default operating technique for adrenalectomy. However, in case of large mass or left side tumor, TPA was preferred in early period. The indications for PRA has been widened over time, TPA is now performed only in large tumor $>6 \mathrm{~cm}$. All operations were performed by 5 experienced endocrine surgeons.

\section{Operation methods}

The surgical techniques for TPA and PRA were same way as previously published studies in our institution (12). Briefly, TPA is performed with a patient positioned in a lateral decubitus position, while operation bed is flexed at the level of the iliac crest. Three accesses are made at $2 \mathrm{~cm}$ below subcostal margin regardless of the right or left adrenal gland. For the left adrenal gland, the spleen and pancreatic tail are mobilized, and for the right adrenal gland, the right lobe of the liver is mobilized to expose the adrenal gland and inferior vena cava. Adrenal gland is then detached from the kidney and retroperitoneal soft tissue. The central vein of the adrenal gland is usually clipped and all feeding vessels are ligated with energy device and divided. The adrenal gland is then retrieved.

In PRA, patient is placed prone in the jackknife position. Three accesses are made below the lowest tip of the $12^{\text {th }}$ rib. After creating the working space using $\mathrm{CO}_{2}$ insufflation, Gerota's fascia is opened, and the kidney upper pole is mobilized to expose the adrenal gland. Adrenal gland is dissected from surrounding adipose tissue. After medial isolation of the adrenal central vein, the vessel is ligated. The gland is placed in an endopouch and retrieved.

\section{Outcomes}

Estimated blood loss was calculated by subtracting the amount of saline used to irrigation from the total volume drained within the suction bottle, after the operation. Gauze was not used during laparoscopic operation.

Regular oral NSAIDs were routinely prescribed 


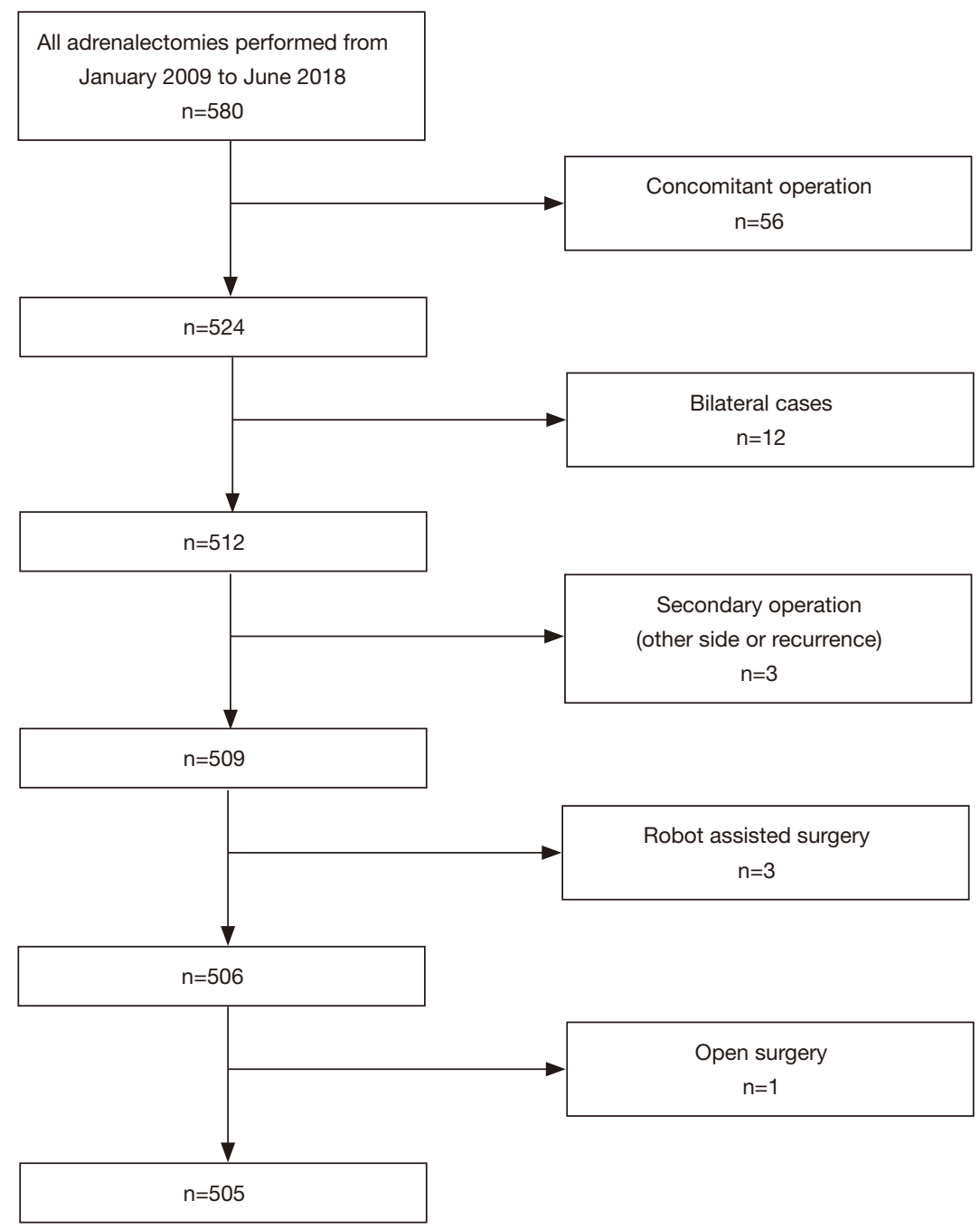

Figure 1 Flow chart of inclusion of the study sample.

to patients during hospitalization. In case that patient complained of numerical rating scale (NRS) $>4$ pain despite taking oral analgesics, 1 ample of intravascular (IV) or intramuscular (IM) pethidine was prescribed. The method of injecting analgesics was determined by whether the patient had previous IV line or not. Pain was re-evaluated 30 minutes to 1 hour after injection. If the pain had not been subsided after the pethidine injection, 1 ample of IV or IM tramadol was prescribed alternately. The minimum interval for each drug was 4 hours. The number of pethidine and tramadol usage was analyzed in this study.

Abdominal X-ray or CT were not routinely checked after surgery. Patients were allowed to drink 2 hours after surgery and to eat soft diet from the evening of the day of surgery, either in TPA or PRA cases. In case of difficult operation due to adhesion or large mass, patients got their first meal on the first day after surgery. The difficulty of the operation was determined by the operator, generally based on the 'laparoscopic' operation time $>1$ hour.

Data were described as 'mean, range' except for operative time (mean \pm standard deviation).

\section{Statistical analysis}

Chi-squared and Fisher's exact tests were used to compare categorical data. For continuous data, analysis of variance and Kruskal-Wallis tests were used. All data were analysed with IBM SPSS Statistics ver. 22.0 (IBM, Armonk, NY, USA). A P value $<0.05$ was considered statistically significant. 
Table 1 Baseline characteristics of patients who underwent surgery using the transperitoneal and retroperitoneal approaches

\begin{tabular}{lccc}
\hline & Total & Transperitoneal & Retroperitoneal \\
\hline Number of cases & 505 & 114 & 391 \\
Mean age (years) & $47.57 \pm 12.83$ & $47.73 \pm 13.02$ & $47.53 \pm 12.79$ \\
Sex (male:female) & $188: 377$ & $37: 77$ & $151: 240$ \\
BMl (kg/m ${ }^{2}$ ) & $24.72 \pm 4.06$ & $24.20 \pm 3.58$ & $24.87 \pm 4.18$ \\
Operation side (right:left) & $214: 291$ & $35: 79$ & $179: 212$ \\
Diagnosis (number of cases) & & & 0.092 \\
Hyperaldosteronism & 157 & 25 & 132 \\
Pheochromocytoma & 83 & 29 & 54 \\
Cushing's syndrome & 109 & 15 & $9.004^{*}$ \\
Non-functioning incidentaloma & 128 & 37 & 91 \\
Non-functioning symptomatic tumor & 12 & 5 & 7 \\
Metastatic cancer & 16 & 3 & 13
\end{tabular}

BMI, body mass index. ${ }^{*} \mathrm{P}<0.05$.

\section{Ethical statement}

The study was conducted in accordance with the Declaration of Helsinki (as revised in 2013). The study was approved by the institutional review board (IRB) of the Yonsei University College of Medicine (IRB No. 4-2020-1260). Informed consent was waived due to the retrospective nature of the study.

\section{Results}

Table 1 lists the baseline characteristics of the patients. An overview of the characteristics showed smaller tumors tended to be treated with PRA rather than TPA $(\mathrm{P}=0.000)$.

The comparison of outcomes between TPA and PRA is demonstrated in Table 2. Intraoperative factors that were included in the analysis were operative time and estimated blood loss. Operative time was significantly shorter with PRA than with TPA (TPA: $117.02 \pm 54.98 \mathrm{~min}$, PRA: $83.65 \pm 31.22 \mathrm{~min}$; $\mathrm{P}=0.000)$; however, there was no significant difference in the estimated blood loss.

Time to postoperative diet initiation (TPA: $0.85 \pm 0.76$ days, PRA: $0.34 \pm 0.58$ days; $\mathrm{P}=0.000)$ and discharge (TPA: $4.96 \pm 2.74$ days, PRA: $3.55 \pm 1.98$ days; $\mathrm{P}=0.000)$ were significantly shorter in patients who underwent PRA. They also used postoperative analgesic medication less frequently (TPA: $2.90 \pm 2.71$ times, PRA:
$2.35 \pm 2.20$ times; $\mathrm{P}=0.048)$. There was no significant difference in the number of cases involving conversion to other methods; there was one case of conversion from TPA to open surgery and three cases of conversion from PRA to open surgery $(\mathrm{P}=0.642)$. In the TPA case, conversion was due to inferior vena cava injury during the operation. In the PRA cases, conversions were due to splenic artery injury, tumor invasion, and severe adhesions caused by previous radiotherapy, respectively. There was also no significant difference in the number of postoperative complications (TPA: 0, PRA: $1 ; \mathrm{P}=0.774)$. In one case after PRA, postoperative temporary creatinine elevation with slight urine output decrease was noted. Patient had type 2 diabetes but no diabetes related renal disease. There were no kidney exposure or physical injury to renal parenchyme during operation. The patient had been recovered after hydration and conservative management.

Additional analyses for the comparison of outcomes classified by time intervals with ad hoc analysis are shown in Tables 3,4. Sub-groups 1, 2, and 3 comprised PRA cases from 2009-2012, 2013-2015, and 2016-2018, respectively. The time to postoperative diet initiation shortened over time (Sub-group 1: $0.57 \pm 0.56$ days, Sub-group 2: $0.43 \pm 0.72$ days, and Sub-group 3: $0.19 \pm 0.44$ days; $\mathrm{P}=0.000)$. Although the postoperative length of hospital stay showed significant differences among the three 
Table 2 Comparison of outcomes between the transperitoneal and retroperitoneal approaches (including open converted cases)

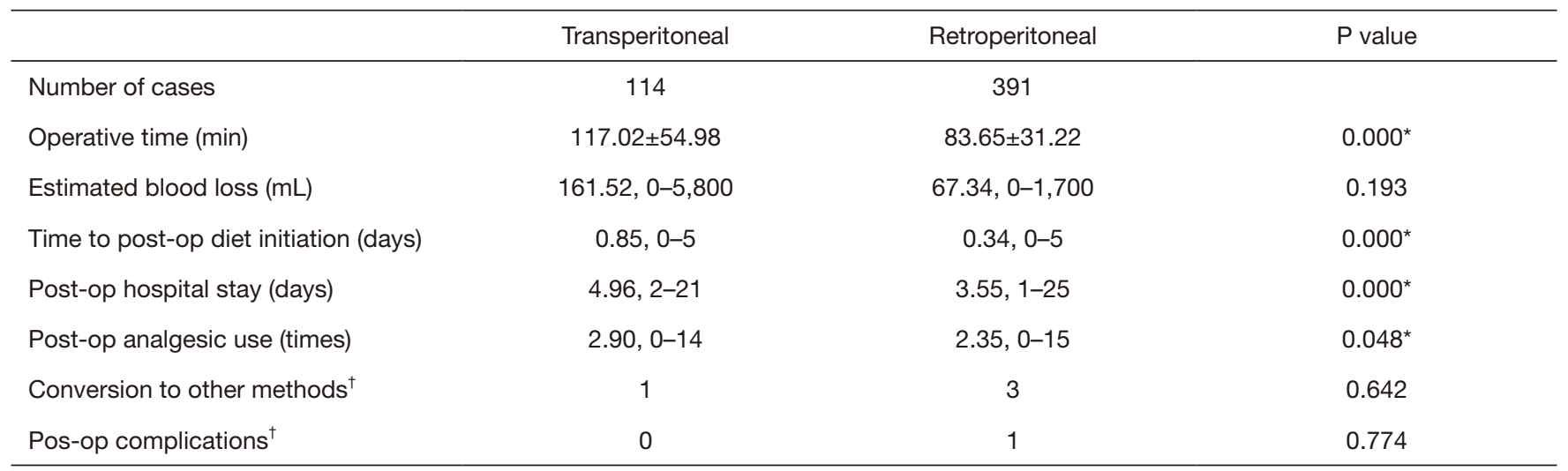

${ }^{\dagger}$ Fisher's exact test; * $\mathrm{P}<0.05$. Post-op, postoperative.

Table 3 Trends in the outcomes of the retroperitoneal approach (including open converted cases)

\begin{tabular}{|c|c|c|c|c|}
\hline & 2009-2012 & 2013-2015 & 2016-2018 & $P$ value \\
\hline Operative time (min) & $83.59 \pm 25.64$ & $83.34 \pm 34.26$ & $83.86 \pm 31.93$ & 0.990 \\
\hline Estimated blood loss (mL) & $180.44,0-1,700$ & $34.65,0-900$ & $32.86,0-1,350$ & 0.212 \\
\hline Time to post-op diet initiation & $0.57,0-2$ & $0.43,0-5$ & $0.19,0-3$ & $0.000^{*}$ \\
\hline Post-op analgesic use (times) & $1.56,0-10$ & $2.35,0-10$ & $2.72,0-15$ & $0.000^{*}$ \\
\hline
\end{tabular}

${ }^{*} \mathrm{P}<0.05$.

Table 4 Trends in the outcomes of the retroperitoneal approach, post-hoc analysis

\begin{tabular}{lc}
\hline Post-hoc analysis (Games-Howell test) & P value \\
\hline Time to post-op diet initiation & \\
2009-2012 vs. 2013-2015 & 0.227 \\
2016-2018 & 0.000 \\
2013-2015 vs. 2016-2018 & 0.006 \\
Post-op hospital stay & \\
2009-2012 vs. 2013-2015 & 0.021 \\
2016-2018 & 0.927 \\
2013-2015 vs. 2016-2018 & 0.015 \\
Post-op analgesic use & \\
2009-2012 vs. 2013-2015 & 0.026 \\
2016-2018 & 0.000 \\
2013-2015 vs. 2016-2018 & 0.329 \\
\hline
\end{tabular}

Post-op, postoperative. sub-groups, the findings were not specific for a longer or shorter duration in the ad hoc analysis (Sub-group 1: $3.30 \pm 1.50$ days, Sub-group 2: $4.05 \pm 3.015$ days, and Sub-group 3: $3.55 \pm 1.976$ days). Postoperative analgesic use increased over time with statistical significance (Sub-group 1: $1.56 \pm 1.75$ times, Sub-group 2: 2.35 \pm 2.11 times, and Sub-group 3: $2.72 \pm 2.35$ times; $\mathrm{P}=0.000$ ).

Changes in surgical case selection over time are demonstrated in Table 5 by the comparison of PRA cases. Tumor size, operation side, and pathologic results were analyzed. The number of left side adrenalectomy and metastatic cancer has been increased at latter stage, but there was no statistically significant differences.

\section{Discussion}

In this study, the comparison of surgical outcomes between PRA and TPA showed that PRA was associated with a shorter operative time, earlier diet initiation, shorter 
Table 5 Retroperitoneal approach: surgical difficulty comparison (including open converted cases)

\begin{tabular}{lccc}
\hline & $2009-2012$ & $2013-2015$ & $2016-2018$ \\
\hline Number of cases & 90 & 110 & 191 \\
Operation side (right:left) & $46: 44$ & $56: 54$ & $77: 114$ \\
Diagnosis (number of cases) ${ }^{\dagger}$ & & & 0.106 \\
Hyperaldosteronism & 30 & 38 & 64 \\
Pheochromocytoma & 14 & 20 & 20 \\
Cushing's syndrome & 18 & 24 & 52 \\
Non-functioning incidentaloma & 23 & 19 & 49 \\
Non-functioning symptomatic tumor & 4 & 1 & 2 \\
Metastatic cancer & 1 & $2.64 \pm 1.48$ & 4 \\
Tumor size (cm) & $2.78 \pm 1.54$ & & $2.61 \pm 1.55$ \\
\hline
\end{tabular}

${ }^{\dagger}$ Fisher's exact test.

hospital stay, and less frequent analgesic use, without compromising patient safety in terms of open conversion or complication rates. The findings also suggest that better outcomes, in terms of earlier diet initiation, are feasible with accumulation of surgical experience.

Laparoscopic adrenalectomy has become a standard method for treating various adrenal lesions. It has overcome the disadvantages of conventional open surgery, such as long surgical incisions and prolonged hospital stay (3). Although open adrenalectomy should be considered for treating adrenal tumors larger than $8 \mathrm{~cm}$, tumors with malignant features such as local or vascular invasion, recurrent disease, and emergency cases $(13,14)$, the indication for laparoscopic surgery has been expanded with the development of newer instruments and surgical techniques. There are many reports of successful laparoscopic removal of large adrenal masses (15-17). Our center has reported excellent surgical outcomes of laparoscopic adrenalectomy for the management of primary hyperaldosteronism and pheochromocytoma, consistent with the findings of other previous studies $(18,19)$.

There are various laparoscopic approaches to adrenalectomy, including the transperitoneal, lateral retroperitoneal, posterior retroperitoneal, and transthoracic approaches $(10,20,21)$. Among these, the transperitoneal and posterior retroperitoneal approaches are the most common techniques studied. TPA has gained popularity as it offers many advantages such as low morbidity, short hospitalization, improved cosmesis, and rapid recovery, while providing a large and familiar operating field $(8,11)$.
It also permits concomitant operations for intra-abdominal lesions and quick conversion to open surgery in difficult or emergent situations (22). However, its shortcomings include the possibility of intraoperative intra-abdominal organ injury and postoperative adhesions.

Although the operating field for PRA is narrower than that for TPA and the retroperitoneal anatomy is tricky, PRA provides direct and rapid access to the adrenal glands without the need to mobilize the bowels (10). This reduces postoperative ileus and bacterial contamination (23) and results in a shorter operative time, shorter hospital stay, and faster oral intake resumption than those with TPA $(11,24)$. Although PRA is technically challenging because of the unfamiliar operating field (owing to the reverse position of the patient), Barczyński et al. reported that this can be overcome by gaining experience with $20-25$ cases to flatten the learning curve (25). Several factors may influence case selection for PRA. First, larger tumors are more difficult to remove. Second, left adrenalectomy is traditionally considered more difficult as the gland is positioned beyond the upper pole of the kidney anteriorly. Third, the resection of tumors such as pheochromocytomas is tricky due to risks of catecholamine secretion and bleeding.

In our study, it was found that the difference in operating time over time was not significant. Authors concluded that learning curve might have been flattened during first period with 90 cases. However, more difficult cases like left side adrenalectomy or metastatic cancer have been included more in later stage since the indications for PRA have been gradually expanded in our center. This may have resulted 
in no difference in average operation time between three periods.

Although the complication or conversion to open surgery rate were very low in our constitution, there were three open conversions during PRA. The first one was due to splenic artery injury. Since left adrenal gland is located medially to the spleen and superior to the splenic artery and vein, and lateral to the abdominal aorta, cautious manipulation is required during dissection. In case of obese patients, adipose tissue may block the blood vessels to recognize. Excessive dissection to ventral and inferior direction can injure splenic vessels. Second case was converted to open surgery due to metastatic pancreatic cancer to adrenal gland with wide invasion to adjacent soft tissue. PRA was planned as preoperative imaging study showed small solitary adrenal metastasis, with sufficient consultation with the patient about the possibility of laparotomy. Third patient had severe adhesions to the operating field due to previous radiotherapy which had been done for hepatocellular carcinoma (HCC). Although adrenal metastasis was found at contralateral (left) side, dense fibrous adhesion interfered operation field. In all cases, patients were converted to the open methods and recovered well after surgery.

The one complication case after PRA was temporary creatinine elevation. This can happen due to the intraoperative renal manipulation or renal injury. However, it might related to dehydration and drugs used during anesthesia and operation. Close monitoring for renal function after surgery especially in case of known renal disease or diabetic patients is necessary.

Currently, all adrenalectomies are performed using the posterior retroperitoneal approach in our center, except for large tumor $>6 \mathrm{~cm}$ or malignant tumor suspected of invading the surrounding organs. However, patient's past history and imaging results should be carefully reviewed when planning surgery. This study adds to previous findings that the feasibility and safety of PRA are comparable to those of conventional TPA. It also suggests that surgical competence can be achieved over time, improving patient outcomes.

Our study has several limitations, mainly due to its retrospective design and small sample size. The decision for diet initiation was based on the operator's experience, without an objective examination for bowel movement. The indications for PRA has been changed over time, tumor size and pathologic results were not controlled between subgroups. Because of the small sample size, a few outlier can affect the trend of the overall statistic. The hospital stay of our PRA cohort was longest during 2009-2012. According to our raw data, patients with metastatic carcinoma which has been operated mostly in this period had longer hospital stay than other cases. In the last period, the number of analgesic use was highest. Three among the four of the open converted operation cases were included at this period. One IVC injury during TPA for $8 \mathrm{~cm}$ sized pheochromocytoma and two with malignant invasion and adhesion were those. These patients complained more postoperative pain due to laparotomy wound. This may be interpreted as an inevitable results as the number of operations with higher difficulty increases over time. This study included a simple comparison, and in-depth analysis can be carried out in future studies using the CUSUM learning curve. For better analysis about postoperative analgesic usage, patient surveys using pain scales, such as numerical rating scale or visual analog scale, can be incorporated in future research. However, this single center study can give additional information to the debate about the advances of PRA vs. TPA.

\section{Conclusions}

In conclusion, PRA is a favorable surgical method that results in similar patient outcomes compared to those of conventional TPA without compromising patient safety. Surgical competence can be achieved early. More research is required to evaluate pain from the patients' perspective and the learning curve from the surgeons' perspective.

\section{Acknowledgments}

Funding: None.

\section{Footnote}

Reporting Checklist: The authors have completed the STROBE reporting checklist. Available at https://dx.doi. org/10.21037/gs-21-178

Data Sharing Statement: Available at https://dx.doi. org/10.21037/gs-21-178

Conflicts of Interest: All authors have completed the ICMJE uniform disclosure form (available at https://dx.doi. org/10.21037/gs-21-178). The authors have no conflicts of interest to declare. 
Ethical Statement: The authors are accountable for all aspects of the work in ensuring that questions related to the accuracy or integrity of any part of the work are appropriately investigated and resolved. The study was conducted in accordance with the Declaration of Helsinki (as revised in 2013). The study was approved by the institutional review board (IRB) of the Yonsei University College of Medicine (IRB No. 4-2020-1260), and individual informed consent for this retrospective analysis was waived.

Open Access Statement: This is an Open Access article distributed in accordance with the Creative Commons Attribution-NonCommercial-NoDerivs 4.0 International License (CC BY-NC-ND 4.0), which permits the noncommercial replication and distribution of the article with the strict proviso that no changes or edits are made and the original work is properly cited (including links to both the formal publication through the relevant DOI and the license). See: https://creativecommons.org/licenses/by-nc-nd/4.0/.

\section{References}

1. Gagner M, Lacroix A, Bolté E. Laparoscopic adrenalectomy in Cushing's syndrome and pheochromocytoma. N Engl J Med 1992;327:1033.

2. Assalia A, Gagner M. Laparoscopic adrenalectomy. Br J Surg 2004;91:1259-74.

3. Brunt LM, Doherty GM, Norton JA, et al. Laparoscopic adrenalectomy compared to open adrenalectomy for benign adrenal neoplasms. J Am Coll Surg 1996;183:1-10.

4. Nicolai N. Laparoscopic adrenalectomy. Tumori 2003;89:556-9.

5. Prinz RA. A comparison of laparoscopic and open adrenalectomies. Arch Surg 1995;130:489-92; discussion 92-4.

6. Zacharias M, Haese A, Jurczok A, et al. Transperitoneal laparoscopic adrenalectomy: outline of the preoperative management, surgical approach, and outcome. Eur Urol 2006;49:448-59.

7. Gill IS. Needlescopic urology: current status. Urol Clin North Am 2001;28:71-83.

8. Rubinstein M, Gill IS, Aron M, et al. Prospective, randomized comparison of transperitoneal versus retroperitoneal laparoscopic adrenalectomy. J Urol 2005;174:442-5; discussion 445.

9. Walz MK, Peitgen K, Krause U, et al. [Dorsal retroperitoneoscopic adrenalectomy--a new surgical technique]. Zentralbl Chir 1995;120:53-8.
10. Li QY, Li F. Laparoscopic adrenalectomy in pheochromocytoma: retroperitoneal approach versus transperitoneal approach. J Endourol 2010;24:1441-5.

11. Mohammadi-Fallah MR, Mehdizadeh A, Badalzadeh A, et al. Comparison of transperitoneal versus retroperitoneal laparoscopic adrenalectomy in a prospective randomized study. J Laparoendosc Adv Surg Tech A 2013;23:362-6.

12. Lee CR, Walz MK, Park S, et al. A comparative study of the transperitoneal and posterior retroperitoneal approaches for laparoscopic adrenalectomy for adrenal tumors. Ann Surg Oncol 2012;19:2629-34.

13. Tiyadath BN, Sukumar S, Saheed CS, et al. Laparoscopic adrenalectomy --- is it any different in phaeochromocytoma and non-phaeochromocytoma? Asian J Surg 2007;30:244-9.

14. Taffurelli G, Ricci C, Casadei R, et al. Open adrenalectomy in the era of laparoscopic surgery: a review. Updates Surg 2017;69:135-43.

15. MacGillivray DC, Whalen GF, Malchoff CD, et al. Laparoscopic resection of large adrenal tumors. Ann Surg Oncol 2002;9:480-5.

16. Tsuru N, Suzuki K, Ushiyama T, et al. Laparoscopic adrenalectomy for large adrenal tumors. J Endourol 2005;19:537-40.

17. Boylu U, Oommen M, Lee BR, et al. Laparoscopic adrenalectomy for large adrenal masses: pushing the envelope. J Endourol 2009;23:971-5.

18. Kim K, Kim JK, Lee CR, et al. Surgical outcomes of laparoscopic adrenalectomy for primary hyperaldosteronism: 20 years of experience in a single institution. Ann Surg Treat Res 2019;96:223-9.

19. Ban EJ, Yap Z, Kandil E, et al. Hemodynamic stability during adrenalectomy for pheochromocytoma: A case control study of posterior retroperitoneal vs lateral transperitoneal approaches. Medicine (Baltimore) 2020;99:e19104.

20. Del Pizzo JJ. Transabdominal laparoscopic adrenalectomy. Curr Urol Rep 2003;4:81-6.

21. Gill IS, Meraney AM, Thomas JC, et al. Thoracoscopic transdiaphragmatic adrenalectomy: the initial experience. J Urol 2001;165:1875-81.

22. Raffaelli M, De Crea C, Bellantone R. Laparoscopic adrenalectomy. Gland Surg 2019;8:S41-S52.

23. Munch LC, Gill IS, McRoberts JW. Laparoscopic retroperitoneal renal cystectomy. J Urol 1994;151:135-8.

24. Conzo G, Tartaglia E, Gambardella C, et al. Minimally invasive approach for adrenal lesions: Systematic review of laparoscopic versus retroperitoneoscopic adrenalectomy 
and assessment of risk factors for complications. Int J Surg 2016;28 Suppl 1:S118-23.

25. Barczyński M, Konturek A, Golkowski F, et al. Posterior retroperitoneoscopic adrenalectomy: a comparison

Cite this article as: Kook Y, Choi HR, Kang SW, Kim JK, Lee CR, Lee J, Jeong JJ, Nam KH, Chung WY. Laparoscopic adrenalectomy: comparison of outcomes between posterior retroperitoneoscopic and transperitoneal adrenalectomy with 10 years' experience. Gland Surg 2021;10(7):2104-2112. doi: 10.21037/gs-21-178 between the initial experience in the invention phase and introductory phase of the new surgical technique. World J Surg 2007;31:65-71. 